\title{
Analysis of the CARD15 variants R702W, G908R and L1007fs in Italian IBD patients
}

\author{
Daniela Giachino $^{1}$, Marjan Maria van Duist ${ }^{1}$, Silvia Regazzoni ${ }^{1}$, Dario Gregori ${ }^{2}$, \\ Marco Bardessono ${ }^{3}$, Paola Salacone ${ }^{3}$, Nadia Scaglione ${ }^{4}$, Raffaello Sostegni ${ }^{4}$, \\ Nicoletta Sapone ${ }^{5}$, Francesca Bresso ${ }^{5}$, Angela Sambataro ${ }^{3}$, Ezio Gaia ${ }^{3}$, Angelo Pera ${ }^{4}$, \\ Marco Astegiano ${ }^{5}$ and M De Marchi*,1
}

\begin{abstract}
${ }^{1}$ Department of Clinical and Biological Sciences, University of Torino, Italy; ${ }^{2}$ Department of Public Health and Microbiology, University of Torino, Italy; ${ }^{3}$ Gastroenterology Unit, ASO S. Luigi, Orbassano, Italy; ${ }^{4}$ Gastroenterology Unit, Ospedale Mauriziano, Torino, Italy; and ${ }^{5}$ Gastroenterology Unit, ASO San Giovanni Battista, Torino, Italy
\end{abstract}

CARD15 on chromosome 16 is the only IBD susceptibility gene identified among several mapped loci. Its recurrent variants R702W, G908R and L1007fs have shown significant association with Crohn's disease (CD), but not with ulcerative colitis (UC), in different Caucasian populations. We analysed these three variants in $184 \mathrm{CD}$ and 92 UC Italian patients and in 177 healthy controls. L1007fs and G908R were independently associated with $C D$, while $R 702 \mathrm{~W}$ showed a nonsignificant increase. After combining the three variants together, $32.6 \%$ of $C D$ patients were positive vs $18.6 \%$ of the controls. The association was stronger for homozygotes and compound heterozygotes, OR 13.9 (1.8-108), and weaker but still significant for simple heterozygotes, OR 1.7 (1.0-2.9). An excess of homozygotes/compound heterozygotes also resulted from the comparison with Hardy-Weinberg expectations. Phenotypegenotype correlations were analysed first by univariate logistic regression and then by multivariate analysis, the effect of CARD15 positivity being adjusted according to the status of smoking, familiarity and sex, so as to focus on the predictivity of genetic and environmental risk factors on the clinical phenotype. Significant risk estimates of the CARD15 genotype were obtained for stricturing vs inflammatory behaviour, OR 2.76 (1.2-6.3), and for penetrating behaviour, 2.59 (1.0-6.6), and marginally significant for ileal vs colic location, OR 3.0 (0.9-9.8). Our findings indicate that the association of the CARD15 genotype with behaviour and location of disease holds also for the Italian population.

European Journal of Human Genetics (2004) 12, 206-212. doi:10.1038/sj.ejhg.5201130

Published online 28 January 2004

Keywords: CARD15; Crohn's disease; association study

Introduction

Crohn's disease (CD) and ulcerative colitis (UC), the two major forms of inflammatory bowel disease (IBD), display a variety of clinical manifestations and have a multifactorial

${ }^{*}$ Correspondence: Professor M De Marchi, Department of Clinical and Biological Sciences, University of Torino, Regione Gonzole 10, Orbassano 10043, Italy. Tel: + 39011 6708128; Fax: + 39011 9038639;

E-mail: mario.demarchi@unito.it

Received 9 June 2003; accepted 17 October 2003 genetic component. Linkage studies have identified at least eight IBD susceptibility loci on different chromosomes (OMIM \#266 600), some possibly involved in both CD and UC, and some in one disease only. ${ }^{1}$ The aetiology of CD and UC is still unclear. Clinical and experimental evidence, however, points to the involvement of a deregulated natural and immune response to intestinal antigens in their pathogenesis. $^{2}$ The IBD1 locus on chromosome 16 is a major susceptibility locus for $\mathrm{CD},{ }^{3}$ and the only one in which a responsible gene, initially named NOD2 and later 
CARD15, has been identified. ${ }^{4,5}$ It encodes a cytoplasmic protein, mainly expressed in myelomonocytic and dendritic cells, which is known to regulate inflammation and apoptosis through activation of the NF- $\kappa$ B pathway. ${ }^{6}$ The CARD15 protein is also expressed in the intestinal epithelium, where it exerts an antibacterial role. ${ }^{7}$ In Caucasian populations, three recurrent CARD15 variants, namely two missense mutations, R702W and G908R, and the C-terminal 3020insC frameshift mutation leading to L1007fs, have shown a significant association with CD, but not with UC. A previous genetic analysis of Italian patients was confined to the study of L1007fs in CD. ${ }^{8}$ We have now investigated the association of all variants with both CD and UC.

\section{Patients and methods}

In all, 276 unrelated patients, with an established diagnosis of CD (184) or of UC (92), were retrospectively enrolled in three Torino gastroenterology centres: $17 \%$ of the CD patients and $12.5 \%$ of the UC patients were from multiplex families. Clinical features were recorded on a standard form according to the Vienna criteria for $\mathrm{CD}:{ }^{9}$ age at onset (A1 $<40$, A2 $>40$ years), disease behaviour (B1 nonstricturing, nonpenetrating, B2 stricturing, B3 penetrating), location (L1 terminal ileum, L2 colon, L3 ileocolon, L4 upper GI), together with severity, extraintestinal manifestations (rheumatological, dermatological, ocular, liver and biliary, amiloidosis), type of onset and known risk factors such as smoking (yes for current smokers at the time of the study, ex for smokers in the past) and familiarity. The clinical features considered for UC were age at onset, behaviour and extent of the disease, presence of extraintestinal manifestations, familiarity and smoking habits. Diagnoses and all clinical data were validated by the referring clinicians, who were not aware of the CARD15 genotype results. DNA samples from 177 medical students were used as controls. Informed consent was obtained from each of the participants.

\section{Molecular analysis}

The entire sequence of exons 8 and 11 and the relevant segment of the large exon 4 of the CARD15 gene were amplified by PCR using the following primers designed on the published genomic sequence (GenBank CARD15 gene accession number: AJ303140):

\section{Exon 4449 bp product F 5'-AGGTCAGCCTGATGACATTTC-3' R 5'-CGGGATGGAGTGGAAGT-3' \\ Exon 8202 bp product $\mathrm{F}$ 5'-CACTGACACTGTCTGTTGACTC-3' R 5'-AAGACCTTCATAAGTGGCCCC-3' \\ Exon $11217 \mathrm{bp}$ product F 5'-CTGGCTAACTCCTGCAGT-3' R 5'-ACTGAGGTTCGGAGAGCT-3'}

A measure of $100 \mathrm{ng}$ of genomic DNAs was amplified in a final volume of $25 \mu \mathrm{l}$, containing the buffer supplied by the manufacturer, $2 \mathrm{mM} \mathrm{MgCl}_{2}, 10 \mathrm{pmol}$ of each primer,
$50 \mathrm{nmol}$ each dNTP and $1 \mathrm{U}$ AmpliTaq polymerase (Applera). An initial denaturation of $5 \mathrm{~min}$ was followed by 30 cycles of $30 \mathrm{~s}$ at $94^{\circ} \mathrm{C}, 30 \mathrm{~s}$ annealing at $61^{\circ} \mathrm{C}$ for exon 4 , $60^{\circ} \mathrm{C}$ for exon $8,55^{\circ} \mathrm{C}$ for exon 11,30 s elongation at $72^{\circ} \mathrm{C}$, followed by a final extension of $7 \mathrm{~min}$ at $72^{\circ} \mathrm{C}$. The size and amount of the products were checked by agarose gel electrophoresis. The three variants were detected: by digestion of exon 4 with MspI for R702W (NCBI SNP CLUSTER ID: rs2066844), exon 8 with HhaI for G908R (rs2066845) and exon 11 with NlaIV for L1007fs (rs2066847). R702W homozygotes and heterozygotes were discriminated by separation of the small restriction products from exon 4 (wt allele: $329+66+54 \mathrm{bp}$, mutated allele: $329+120 \mathrm{bp}$ ) by $6 \%$ polyacrylamide gel electrophoresis in nondenaturing conditions.

\section{Statistical analysis}

The association of the three CARD15 variants were estimated as odds ratios (OR) and 95\% confidence intervals (CI) through univariate logistic analysis. The genotype frequencies observed in patients and controls were compared with Hardy-Weinberg expectations by using the $\chi^{2}$ statistics. Phenotype-genotype correlations were first analysed by univariate logistic regression and then by multivariate analysis, adjusting the effect of CARD15 positivity according to the status of smoking, familiarity and sex. For all calculations, we used the S-Plus2000 software (Insightful, Inc, USA).

\section{Results}

Genotypes of the three recurrent variants in CD and UC patients and controls are reported in Table 1 . We first evaluated the association with positivity for each variant by grouping homozygous and heterozygous genotypes (Table 2): G908R and L1007fs were significantly more frequent in CD than in controls and UC. The frequency of R702W was also increased, but not significantly, in both $\mathrm{CD}$ and UC; even the conditional comparison of this variant in patient and control subgroups negative for the two other variants only marginally strengthened the association with CD (OR 1.66, CI 0.9-3.0), and not that with UC (OR 1.69, CI 0.8-3.1). In accordance with other studies, we then grouped all three variants into a single 'mutated', M allele, whose gene frequency was $19.6 \%$ in CD, $14.1 \%$ in UC and $9.6 \%$ in controls (Table 3). Again, positivity for this allele was significantly associated with CD only (Table 2). The association with CD was stronger for the $\mathrm{M} / \mathrm{M}$ genotype (OR 13.9), and weaker but still significant for the $\mathrm{M} / \mathrm{wt}$ genotype (OR 1.7, Table 4). Moreover, the $\mathrm{M} / \mathrm{M}, \mathrm{M} / \mathrm{wt}$ and wt/wt genotype frequencies closely approached Hardy-Weinberg expectations among controls, while there was a significant excess of $\mathrm{M} / \mathrm{M}$ in CD. To assess the mutation-phenotype correlations, we first considered the frequency of positivity of CARD 15 
Table 1 Observed CARD15 genotypes

\begin{tabular}{lrrr}
\hline Genotype & $C D(\%)$ & UC (\%) & Controls (\%) \\
\hline L1007fs/L1007fs & $4(2.17)$ & 0 & 0 \\
L1007fs/G908R & $1(0.54)$ & 0 & 0 \\
L1007fs/R702W & $1(0.54)$ & 0 & $1(0.6)$ \\
L1007fs/R702W/R702W & $1(0.54)$ & 0 & 0 \\
L1007fs/wt & $12(6.52)$ & $1(1.09)$ & 0 \\
G908R/R702W & $2(1.09)$ & 0 & $5(3.95)$ \\
G908R/wt & $13(7.06)$ & $5(5.43)$ & 0 \\
R702W/R702W & $3(1.63)$ & $14(15.26)$ & $2.82)$ \\
R702W/wt & $23(12.5)$ & $69(75)$ & $11.3)$ \\
Wt/wt & $124(67.4)$ & 92 & $144(81.35)$ \\
Total & 184 & & 177 \\
\hline
\end{tabular}

${ }^{\mathrm{a}}$ Single instance of L1007fs and R702W in cis on the same haplotype.

Table 2 Association of the recurrent variants with $C D$ and UC

\begin{tabular}{|c|c|c|c|c|c|}
\hline Variant & $C D \%$ & OR $(95 \% \mathrm{Cl})$ & UC (\%) & OR $(95 \% \mathrm{CI})$ & Controls (\%) \\
\hline R702W & 16.3 & $1.6(0.9-3.0)$ & 18.5 & $1.7(0.8-3.0)$ & 11.9 \\
\hline G908R & 8.7 & $3.7(1.3-10.4)$ & 5.4 & $2.1(0.6-7.4)$ & 2.8 \\
\hline L1007fs & 10.3 & $2.8(1.2-6.5)$ & 1.1 & $0.3(0.0-2.1)$ & 4.5 \\
\hline Any variant & 32.6 & $2.4(1.0-5.7)$ & 25 & $1.5(0.8-2.7)$ & 18.6 \\
\hline
\end{tabular}

Table 3 Gene frequencies of the recurrent variants

\begin{tabular}{lccc}
\hline & $\begin{array}{c}\text { CD \% } \\
(n=368)\end{array}$ & $\begin{array}{c}\text { UC (\%) } \\
(n=184)\end{array}$ & $\begin{array}{c}\text { Controls (\%) } \\
(n=354)\end{array}$ \\
\hline R702W & 9.0 & 10.9 & 5.9 \\
G908R & 4.3 & 2.7 & 1.4 \\
L1007fs & 6.3 & 0.5 & 2.3 \\
Any variant (M allele) & 19.6 & 14.1 & 9.6 \\
\hline
\end{tabular}

variants in $\mathrm{CD}$ and UC patients with different clinical features. Univariate analysis disclosed in UC patients no significant association between CARD15 variants and any of the clinical features. In $\mathrm{CD}$, the estimated chance of finding at least one genetic variant was significantly increased among patients with a stricturing behaviour, and showed a marginally significant increase among those with ileal location (Table 5 third column). The same contingency tables were then used to consider each clinical feature (age at onset, behaviour, location, extraintestinal manifestations, type of onset) as an outcome, with sex, familiarity, positivity for a CARD 15 variant and smoking as explanatory variables (Table 5 sixth column). After adjusting CARD15 positivity for smoking, familiarity and sex, multivariate analysis resulted in higher risk estimates for stricturing behaviour and ileal location, with OR 2.76 and 3.0 , respectively; the risk of penetrating behaviour also became significant with OR 2.59.

The statistical significance of most of these correlations vanished when $\mathrm{M} / \mathrm{M}$ and $\mathrm{M} / \mathrm{wt}$ genotypes were considered, and only held for stricturing behaviour (not shown).

\section{Discussion}

This study of the association of the three recurrent CARD15 variants with IBD in Italians found significant associations for L1007fs and G908R with CD only. The

Table 4 CARD15 genotype associations and Hardy-Weinberg disequilibrium in CD

\begin{tabular}{|c|c|c|c|c|c|}
\hline \multirow{2}{*}{ Genotype } & \multicolumn{2}{|c|}{$C D$} & \multicolumn{2}{|c|}{ Controls } & \multirow{2}{*}{ OR $(95 \% C l)^{a}$} \\
\hline & Observed (\%) & Expected $^{b}$ & Observed (\%) & Expected $^{c}$ & \\
\hline $\begin{array}{l}M / M \\
M / w t \\
w t / w t\end{array}$ & $\begin{array}{c}12(6.5) \\
48(26.1) \\
124(67.4) \\
\chi^{2}\end{array}$ & $\begin{array}{r}7.0 \\
58.0 \\
118.9\end{array}$ & $\begin{array}{c}1(0.6) \\
32(18.0) \\
144(81.4) \\
\chi^{2}\end{array}$ & $\begin{array}{r}1.6 \\
30.7 \\
144.6\end{array}$ & $\begin{array}{c}13.9(1.8-108) \\
1.7(1.0-2.9) \\
-\end{array}$ \\
\hline
\end{tabular}

${ }^{\mathrm{a}} \mathrm{CD}$ vs controls.

${ }^{b}$ Expected under Hardy-Weinberg equilibrium with gene frequencies of the $M$ allele 0.195.

${ }^{\mathrm{c}}$ Expected under Hardy-Weinberg equilibrium with gene frequencies of the M allele 0.096.

dObserved $v s$ expected. 
Table 5 Genotype-phenotype correlations

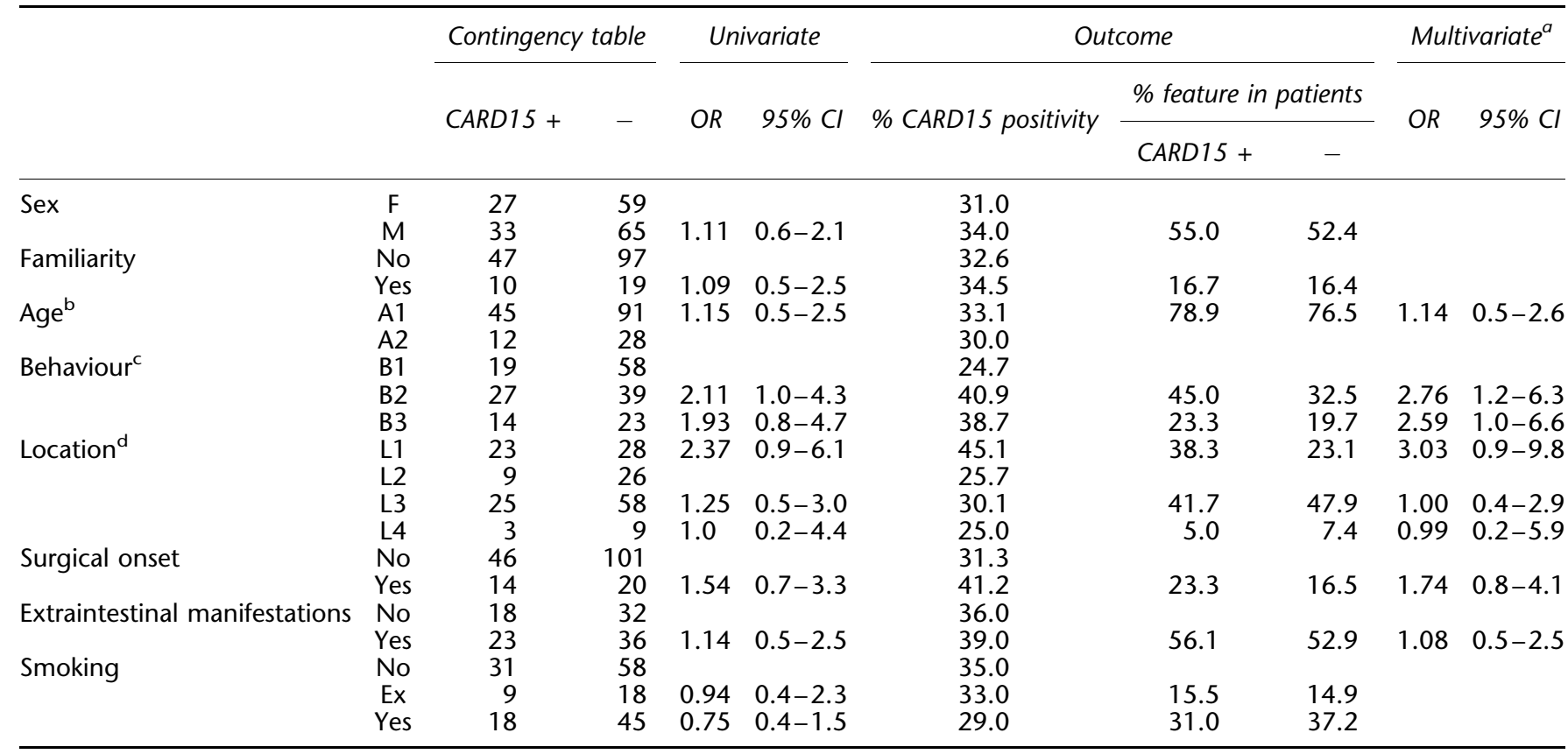

${ }^{\mathrm{a}} \mathrm{OR}$ adjusted for sex, familiarity and smoking, whose OR are not shown.

${ }^{\mathrm{b}} \mathrm{A} 1<40, \mathrm{~A} 2>40$ years.

CB1 nonstricturing, nonpenetrating, B2 stricturing, B3 penetrating.

dL1 terminal ileum, L2 colon, L3 ileocolon, L4 upper Gl.

frequency of R702W was slightly but not significantly increased in both CD and UC patients $v s$ controls. Since a previous study in Italy only considered L1007fs, ${ }^{8}$ these findings provide the first confirmation in Italian patients of the CARD15/CD association reported in other Caucasian populations (Table 6). The overall fraction of CD patients with at least one variant was $32.5 \%$, lower than in Quebec ${ }^{10}$ and western Europe, ${ }^{11}$ but similar to that in Germany and the UK, ${ }^{12}$ and higher than in Ireland ${ }^{13}$ and Crete. ${ }^{14}$ In most studies, the L1007fs variant displayed the strongest association, while those of G908R and R702W were weaker and only significant in some studies. This may result both from the limited number of patients (ranging from 55 to 688) or controls (62-409), as well as from true ethnic differences. As noted by Hirschhorn et $a l^{15}$ and Colhoun et al, ${ }^{16}$ replication of weaker associations, even if true, is difficult, and requires larger samples. In this respect, the case of CARD15 variants in CD is a robust exception if compared with all previous IBD association studies, which considered several polymorphisms, for example, in the TNF $\alpha$ promoter, IL-1 and other cytokine genes and receptors, and led to highly variable findings. ${ }^{1}$ An extreme example of an ethnic difference is the lack of the three variants repeatedly reported in CD and the general population of Japan ${ }^{17-19}$ and Korea. ${ }^{20}$ Since, in these studies, CARD15 has been extensively resequenced in CD patients, any role of IBD1 is unlikely to depend on other coding sequence variants.
Other IBD susceptibility loci are probably responsible for CD susceptibility in those Eastern populations. In Ashkenazi Jews, a population with high incidence of $C D$, the CARD15 gene is involved, but with a peculiar allelic spectrum encompassing G908R and L1007fs, but not R702W. ${ }^{21}$ Interestingly, an additional, population-restricted risk haplotype was present in a substantial fraction of these patients, but no pathogenic mutation(s) could be identified. ${ }^{21}$ In the survey conducted by Lesage et $a l^{11}$ on 453 European CD patients, the fraction of positive patients increased from 41.5 to $49.4 \%$, thanks to an extensive search of rarer variants in the entire coding sequence in addition to the three recurrent ones. Similarly, to fully estimate the contribution of CARD15 to CD susceptibility, it would also be necessary to characterize rare or population-restricted variants in Italians. In other populations, stronger associations have been reported for homozygotes and compound heterozygotes (OR values of 20-40) than for simple heterozygotes (OR 2-4). ${ }^{1}$ Our study is in agreement with such a gene-dosage effect, although at lower levels - OR 13.9 and 1.7, respectively. An excess of patients carrying two CARD15 variant alleles also resulted from comparison with Hardy-Weinberg expectations, a kind of analysis that may overcome limitations originating from the rarity of some genotypes in the control group. The excess of homozygotes and compound heterozygotes suggests a multiplicative rather than additive action of the 
Table 6 Association of CD with recurrent CARD15/NOD2 variants in different populations

\begin{tabular}{|c|c|c|c|c|c|}
\hline \multicolumn{3}{|c|}{ Gene frequency } & \multirow{3}{*}{$\begin{array}{c}\% \text { Overall positivity } \\
P / C\end{array}$} & \multirow{3}{*}{$\begin{array}{c}\text { Sample size } \\
\text { P/C }\end{array}$} & \multirow{3}{*}{ Population ${ }^{\text {Ref. }}$} \\
\hline$R 702 \mathrm{~W}$ & G908R & L1007fs & & & \\
\hline$P / C^{a}$ & $P / C$ & $P / C$ & & & \\
\hline 12.9 & 5.2 & 10.3 & 45.0 & 229 & Quebec $^{10}$ \\
\hline 4.2 & 0.7 & 0.7 & 9.0 & 71 & \\
\hline 11.0 & 6.0 & 11.0 & 41.5 & 453 & Several European ${ }^{11,4}$ \\
\hline 4.4 & 1.0 & 1.9 & 14.6 & 103 & \\
\hline 12.5 & 3.3 & 9.4 & 38.5 & 244 & Great Britain 29 \\
\hline 5.2 & 1.4 & 1.6 & 15.8 & 354 & \\
\hline 9.1 & 3.4 & 6.6 & 35.8 & 688 & Germany\&Great Britain ${ }^{12,23}$ \\
\hline 3.5 & 0.6 & 2.1 & 13.6 & 250 & \\
\hline 9.9 & 4.0 & 13.7 & $\mathrm{NG}^{\mathrm{b}}$ & 552 & Germany $^{32}$ \\
\hline 4.8 & 0.7 & 4.1 & & 373 & \\
\hline 3.5 & 0.9 & 1.8 & NG & 55 & Norway $^{32}$ \\
\hline 2.5 & 1.2 & 1.0 & & 202 & \\
\hline 10.7 & 4.3 & 8.4 & NG & 303 & White USA ${ }^{25,5}$ \\
\hline 4.0 & 1.6 & 3.8 & & 288 & \\
\hline 2.6 & 8.7 & 7.3 & NG & 172 & Ashkenazi $^{25,5}$ \\
\hline 4.8 & 3.2 & 1.6 & & $6 \overline{2}$ & \\
\hline 5.1 & 8.3 & 6.1 & 31.8 & 481 & Ashkenazi $^{33}$ \\
\hline 4.5 & 5.5 & 3.6 & & 110 & \\
\hline NG & NG & NG & 30.3 & 112 & Ashkenazi ${ }^{21}$ \\
\hline 11.0 & 2.0 & 7.0 & NG & $\begin{array}{r}19 \\
267\end{array}$ & Australia 34 \\
\hline 5.0 & 1.0 & 1.0 & & 409 & \\
\hline 7.0 & 3.0 & 4.0 & 27.4 & 131 & Ireland $^{13}$ \\
\hline 4.0 & 1.0 & 1.0 & & NG & \\
\hline 3.3 & 0.6 & 4.8 & 15.5 & 271 & Finland $^{35}$ \\
\hline 1.8 & 0.0 & 1.7 & 7.0 & 300 & \\
\hline 0 & 0 & 0 & 0 & 188 & Japan ${ }^{18,} 17$ \\
\hline 0 & 0 & 0 & & 192 & \\
\hline 0.4 & 0 & 0 & 0.8 & 483 & $\operatorname{Japan}^{19}$ \\
\hline 0 & 0 & 0 & 0 & 126 & Korea $^{20}$ \\
\hline 0 & 0 & 0 & & 116 & \\
\hline 9.0 & 4.3 & 6.3 & 32.6 & 184 & Italy this study \\
\hline 5.9 & 1.4 & 2.3 & 18.6 & 177 & \\
\hline - & 4.3 & 8.5 & - & 130 & Netherlands ${ }^{36}$ \\
\hline & 3.0 & 1.0 & & 152 & \\
\hline - & - & 13.9 & - & 97 & Germany $^{37}$ \\
\hline & & 1.7 & & 120 & \\
\hline - & - & 11.7 & - & 133 & Italy ${ }^{8}$ \\
\hline & & 1.2 & & 81 & \\
\hline - & - & 2.7 & - & 56 & Crete $^{14}$ \\
\hline & & 1.5 & & 67 & \\
\hline
\end{tabular}

${ }^{\mathrm{a}} \mathrm{P} / \mathrm{C}$ patients/controls.

${ }^{b} \mathrm{NG}$, not given.

two variants. ${ }^{22}$ Hardy-Weinberg disequilibrium is expected to involve, together with the true disease-susceptibility gene, other neutral nearby loci, if their alleles happen to be associated by linkage disequilibrium. Thus, the different association strength of the three recurrent CARD15 variants may depend on a different linkage disequilibrium with an unknown nearby mutation, that would be stronger for L1007fs and G908R and weaker for R702W. This hypothesis would easily accommodate the finding of IBD1 risk haplotypes with no identified mutation, in both Ashkenazi Jews ${ }^{21}$ and the German population. ${ }^{23}$ However, this hypothesis is now made unlikely by growing evidence of a defective function of the mutated protein in response to pathogen-associated molecular patterns. Ogura et $a l^{24}$ first demonstrated that CARD15 transduces NF- $\kappa \mathrm{B}$ activation signals in transfected epithelial cells in response to lipopolysaccharide, and specifically, as shown later, to its peptidoglycan contaminant. ${ }^{6,25,26}$ Muramyl-dipeptide has been identified as the bacterial component whose response is specifically mediated by CARD15. ${ }^{27}$ These studies demonstrated a defective transducing activity of the three CD-associated variants. CARD15 genotypes with a more severe defect would confer a greater susceptibility, and show stronger disease association, than those with only partially defective function, whose associated risk is indeed weaker. 
The finding of a lower but significant risk of individuals carrying a single variant (heterozygous genotype) could be ascribed to a halved CARD15 dose in their cells.

Alternatively, as postulated by Inohara et al, ${ }^{27}$ even patients typed as heterozygotes may actually lack the CARD15 function because of a second undetected variant on the other allele.

The analysis of mutation-phenotype correlations revealed an increased chance of mutation positivity in patients with stricturing behaviour, and a weaker association with ileal location, in agreement with most previous studies. $^{1}$ However, since ileal location and stricturing behaviour are intertwined and associated with age at onset, familiarity and surgery, ${ }^{28-30}$ these associations are probably influenced by several confounding effects. In a prospective study by a Belgian group, ${ }^{31}$ early development of stricturing or penetrating behaviour was influenced by disease location, clinical activity of the disease, and smoking habit, but not by the CARD15 genotype. In our series, the proportion of familial and sporadic cases and the proportion of patients with smoking habits were largely similar in the groups of patients with CD with or without CARD15 variant. However, when multivariate analysis was performed with ileal location and stricturing and penetrating behaviour as outcomes, and CARD15 positivity as explanatory variable, we found a significant association after weighting for smoking, familiarity and sex. In agreement with other studies, no significant association involved other features, such as type of onset or extraintestinal manifestations.

The three recurrent CARD15 variants are more similar in terms of high prevalence and low conferred risks to gene polymorphisms associated with common multifactorial diseases, than to mutations responsible for Mendelian diseases. As usual in other common diseases, the chances of developing $\mathrm{CD}$ are determined by complex interactions among various involved genes and environmental factors. Identification of such genetic and environmental factors could help to better understand and control the development of inflammatory lesions. Our findings in the Italian population confirm the CARD15 genotype as an explanatory variable to predict the pattern of disease presentation and progression.

\section{Acknowledgements}

We are indebted to all patients for their willingness to participate in the study, nurses for collaboration and Dr I Iliffe for editing the English text. This work was supported by local grants ex60\% to MDM and by 'Fondazione Mauriziana per la ricerca scientifica e per la cura delle malattie infiammatorie croniche intestinali, Compagnia di San Paolo' to $A P$.

\section{References}

1 Bonen DK, Cho JH: The genetics of inflammatory bowel disease. Gastroenterology 2003; 124: 521-536.
2 Fiocchi C: Inflammatory bowel disease: etiology and pathogenesis. Gastroenterology 1998; 115: 182-205.

3 Hugot JP, Laurent-Puig P, Gower-Rousseau C et al: Mapping of a susceptibility locus for Crohn's disease on chromosome 16. Nature 1996; 379: 821-823.

4 Hugot JP, Chamaillard M, Zouali $\mathrm{H}$ et al: Association of NOD2 leucine-rich repeat variants with susceptibility to Crohn's disease. Nature 2001; 411: 599-603.

5 Ogura $\mathrm{Y}$, Bonen DK, Inohara $\mathrm{N}$ et al: A frameshift mutation in NOD2 associated with susceptibility to Crohn's disease. Nature 2001; 411: 603-606.

6 Inohara N, Ogura Y, Nunez G: Nods: a family of cytosolic proteins that regulate the host response to pathogens. Curr Opin Microbiol 2002; 5: 76-80.

7 Jobin C: Intestinal epithelial cells and innate immunity in the intestine: is CARD15/Nod2 another player? Gastroenterology 2003; 124: $1145-1149$.

8 Vavassori P, Borgiani P, D'Apice MR et al: 3020insC mutation within the NOD2 gene in Crohn's disease: frequency and association with clinical pattern in an Italian population. Dig Liver Dis 2002; 34: 153.

9 Gasche C, Scholmerich J, Brynskov J et al: A simple classification of Crohn's disease: report of the Working Party for the World Congresses of Gastroenterology, Vienna 1998. Inflamm Bowel Dis 2000; 6: 8-15.

10 Vermeire S, Wild G, Kocher K et al: CARD15 genetic variation in a Quebec population: prevalence, genotype-phenotype relationship, and haplotype structure. Am J Hum Genet 2002; 71: 74-83.

11 Lesage S, Zouali H, Cezard JP et al: CARD15/NOD2 mutational analysis and genotype-phenotype correlation in 612 patients with inflammatory bowel disease. Am J Hum Genet 2002; 70: 845-857.

12 Cuthbert AP, Fisher SA, Mirza MM et al: The contribution of NOD2 gene mutations to the risk and site of disease in inflammatory bowel disease. Gastroenterology 2002; 122: 867-874.

13 Bairead E, Harmon DL, Curtis AM et al: Association of NOD2 with Crohn's disease in a homogenous Irish population. Eur J Hum Genet 2003; 11: 237-244.

14 Roussomoustakaki M, Koutroubakis I, Vardas EM et al: NOD2 insertion mutation in a Cretan Crohn's disease population. Gastroenterology 2003; 124: 272-273.

15 Hirschhorn JN, Lohmueller K, Byrne E, Hirschhorn K: A comprehensive review of genetic association studies. Genet Med 2002; 4: 45-61.

16 Colhoun HM, McKeigue PM, Davey Smith G: Problems of reporting genetic associations with complex outcomes. Lancet 2003; 361: 865-872.

17 Inoue N, Tamura K, Kinouchi Y et al: Lack of common NOD2 variants in Japanese patients with Crohn's disease. Gastroenterology 2002; 123: 86-91.

18 Sugimura M, Kinouchi Y, Takahashi S et al: CARD15/NOD2 mutational analysis in Japanese patients with Crohn's disease. Clin Genet 2003; 63: 160-162.

19 Yamazaki K, Takazoe M, Tanaka T, Kazumori T, Nakamura Y: Absence of mutation in the NOD2/CARD15 gene among 483 Japanese patients with Crohn's disease. J Hum Genet 2002; 47: $469-472$.

20 Croucher PJ, Mascheretti S, Hampe J et al: Haplotype structure and association to Crohn's disease of CARD15 mutations in two ethnically divergent populations. Eur J Hum Genet 2003; 11: 6-16.

21 Sugimura K, Taylor KD, Lin YC et al: A novel NOD2/CARD15 haplotype conferring risk for Crohn disease in Ashkenazi Jews. Am J Hum Genet 2003; 72: 509-518.

22 Nielsen DM, Ehm MG, Weir BS: Detecting marker-disease association by testing for Hardy-Weinberg disequilibrium at a marker locus. Am J Hum Genet 1998; 63: $1531-1540$ 
23 Hampe J, Cuthbert A, Croucher PJ et al: Association between insertion mutation in NOD2 gene and Crohn's disease in German and British populations. Lancet 2001; 357: $1925-1928$.

24 Ogura Y, Inohara N, Benito A, Chen FF, Yamaoka S, Nunez G: Nod2, a Nod1/Apaf-1 family member that is restricted to monocytes and activates NF-kappaB. J Biol Chem 2001; 276: $4812-4818$.

25 Bonen DK, Ogura Y, Nicolae DL et al: Crohn's disease-associated NOD2 variants share a signaling defect in response to lipopolysaccharide and peptidoglycan. Gastroenterology 2003; 124: $140-146$.

26 Chamaillard M, Philpott D, Girardin SE et al: Gene-environment interaction modulated by allelic heterogeneity in inflammatory diseases. Proc Natl Acad Sci USA 2003; 100: 3455-3460.

27 Inohara N, Ogura Y, Fontalba A et al: Host recognition of bacterial muramyl dipeptide mediated through NOD2. Implications for Crohn's disease. I Biol Chem 2003; 278: $5509-5512$.

28 Polito JM, Childs B, Mellits ED, Tokayer AZ, Harris ML, Bayless TM: Crohn disease: influence of age at diagnosis on site and clinical type of disease. Gastroenterology 1996; 111: $813-815$.

29 Ahmad T, Armuzzi A, Bunce $\mathrm{M}$ et al: The molecular classification of the clinical manifestations of Crohn's disease. Gastroenterology 2002; 122: 854-866.
30 Abreu MT, Taylor KD, Lin YC et al: Mutations in NOD2 are associated with fibrostenosing disease in patients with Crohn's disease. Gastroenterology 2002; 123: 679-688.

31 Louis E, Michel V, Hugot JP et al: Early development of stricturing or penetrating pattern in Crohn's disease is influenced by disease location, number of flares, and smoking but not by NOD2/ CARD15 genotype. Gut 2003; 52: 552-557.

32 Hampe J, Frenzel H, Mirza MM et al: Evidence for a NOD2independent susceptibility locus for inflammatory bowel disease on chromosome 16p. Proc Natl Acad Sci USA 2002; 99: 321-326.

33 Zhou Z, Lin XY, Akolkar PN et al: Variation at NOD2/CARD15 in familial and sporadic cases of Crohn's disease in the Ashkenazi Jewish population. Am J Gastroenterol 2002; 97: 3095-3101.

34 Cavanaugh JA, Adams KE, Quak EJ et al: CARD15/NOD2 risk alleles in the development of Crohn's disease in the Australian population. Ann Hum Genet 2003; 67: 35-41.

35 Helio T, Halme L, Lappalainen $M$ et al: CARD15/NOD2 gene variants are associated with familially occurring and complicated forms of Crohn's disease. Gut 2003; 52: 558-562.

36 Murillo L, Crusius JB, van Bodegraven AA, Alizadeh BZ, Pena AS: CARD15 gene and the classification of Crohn's disease. Immunogenetics 2002; 54: 59-61.

37 Radlmayr M, Torok HP, Martin K, Folwaczny C: The C-insertion mutation of the NOD2 gene is associated with fistulizing and fibrostenotic phenotypes in Crohn's disease. Gastroenterology 2002; 122: 2091-2092. 